\title{
Understanding domestic social media use among Chinese college students under the framework of uses and gratifications
}

\author{
Hua Pang, TU Dresden, Institute of Media and Communication \\ cassieph@163.com
}

\begin{abstract}
Although China has the world's largest population of social media users, little is known what drives Chinese users to adopt the country's leading media platforms, like QQ, WeChat, and Weibo, and what gratifications are satisfied by using these fastest-growing sites. In light of the literature on the uses and gratifications theory, the study explored the essential pattern of computer-mediated communication phenomena and interaction behaviors in Mainland China. In this exploratory study, 258 college students from Chinese universities were asked about their uses and gratifications of these social media sites. The factor analysis reveals five key dimensions relating to gratifications obtained from social media are identified: relationship maintenance, amusement, style, information seeking and sociability. Moreover, a hierarchical OLS regression analysis shows that there is a positive relationship between frequency of social media use and the needs of relationship maintenance and amusement. Furthermore, among the five socio-psychological values, the social related factor is revealed to be significantly and positively linked with spending time on the social media.
\end{abstract}

Keywords

Social media, uses and gratifications, gratifications sought, gratifications obtained, college students, China

\section{Introduction}

Over the past five years, the online phenomenon of social media has become increasingly prevalent in Chinese people's daily life. According to government data from the China Internet Network Information Center (CNNIC), there were approximately 659 million Chinese SNS users by the end of June 2015 (CNNIC, 2015). Recent research has also shown that almost $98 \%$ of Internet users and $48 \%$ of the total Chinese population have at least one social media account (Verot, 2016). Thus it can be seen that although the Chinese government strongly controls and censors Internet activities, many citizens nevertheless extensively use these novel technologies. The blocking of global networks such as Facebook, Twitter and YouTube in Mainland China has led to the flourishing of domestic social media platforms dominated by the top three players consisting of QQ, Weibo, and WeChat (Linkfluence, 2016). Dominating today's media-centered lifestyle, these local favourite social networking applications are substantially transforming the way in which Chinese people communicate, share news as well as maintain relationships with one another (Cheng, Liang, \& Leung, 2015; Leung, 2013; Zhang \& Pentina, 2012; Zhong, 2014).

Social networking sites (SNSs) are web-based services that offer unprecedented opportunities for users to conveniently browse, post, and comment on diversified content by mutual interests (Choi, 2016; Kammerl \& Kramer, 2016; Lin, Hsu, Chen, \& Fang, 2017). Uses and gratifications (U\&G) research have begun to investigate what the underlying structure of motivates, use behavior and psychological satisfaction regarding the use of new communication tools. Much effort and research have adopted this framework to determine a broad range of psychological motives driving the utilization of different online websites, including Youtube, MySpace and Facebook (Malik, Dhir, \& Nieminen, 2016; Phua, Jin, \& Kim, 2017; Ruehl \& Ingenhoff, 2015). However, this line of studies overemphasizing the gratification 
sought behind the use of social media, neglecting of the investigation of users' gratification obtained practice in the use of these emerging new media. Moreover, scholarly research addressing psychological motivations and use patterns of social media have been predominately conducted in the western countries (Ifinedo, 2016; Phua, Jin, \& Kim, 2017; Quinn, 2016).

Unfortunately, to date, little research has examined these issues for domestic social media platforms in Mainland China (Ji, 2017). Furthermore, prior studies have showed that research on Chinses social media motivations and use patterns is still in the early stage, currently, the issue remains theoretically underdeveloped and empirically unexplored (Ji, 2017). Given the rapid rise of these local social media technologies and their potential use in many fields such as political policy, civic participation, and business in the Chinese society, the fundamental questions warrant research: Why and how people actively employ these technologies? Furthermore, are they fulfilled by the satisfy the certain social or psychological needs through the medium? These intriguing questions are extremely important to today's online media researchers, practitioners as well as policy-makers.

Hence, the objective of the exploratory study is to address the aforementioned shortcomings in the past research by applying the U\&G theory to examine the gratifications that young people seek and the gratifications received from the ongoing use of the top three popular social networks - QQ, WeChat, and Weibo in a given social setting. In line with Rosengren's framework of the U\&G approach (Rosengren, 1974), the research not only reports on the more personal psychological factors but also elaborates on the societal environment and media use structures. According to Rosengren, certain communication needs, individual differences, as well as contextual societal factors can combine to result in different perceived motivations for media use (Rosengren, 1974). This also supports the notion that the explanatory potential of the proposed resaech model needs embracing the ques- tion of the etiology components of media use motivations (Vorderer, Steen, \& Chan, 2006). Thus, this article may not only foster a better understanding of domestic social media phenomenon and its far-reaching implications in the context of China, but provide insights into how people incorporate the three emerging technologies into their daily lives.

\section{Understanding social media use in Mainland China}

Nowadays, China has the largest and fast-developing active virtual space in the world, with a staggering of more than 500 million projected users until 2018 (Statista, 2015).

QQ, WeChat and Weibo are regarded as the leading social networking websites in China, now with 850 million, 600 million and 287 million active monthly users respectively (Linkfluence, 2016). QQ as one of the most popular instant messaging platforms, which constitutes the largest online community in Mainland China, bundled with different services such as online gaming, shopping, microblogging, group chatting and social networking (Huang, Kim, \& Kim, 2013). Weibo is a Twitter-like microblogging platform that enables individuals to publicly post short tweets or messages within 140 characters to friends' webpages immediately, ensuring timely updates of the current activities, ideas, and state in real time (Hu, Marchiori, Kalbaska, \& Cantoni, 2014; Sullivan, 2014; Zhang \& Pentina, 2012). WeChat is a form of mobile messaging app that provides users with a convenient way to exchange text and voice messages, to share ideas and location quickly (Gan, 2017).

Social media use has a special relevance for university students, who are mostly heavy users of the favorite communication service (Zhong, 2014). Previous studies have also indicated that educated youth adults especially students feels more comfortable with the Internet than do the elderly, hence they would prefer to be online consumers and users of different channels of social networking sites 
(Ku, Chu, \& Tseng, 2013; Leung, 2013; Ragnedda, 2015). More recently, a vast volume of literature have shown that the student cohort is dedicated, engaged, and highly motivated to spend time and energy in sharing information, listening to music, uploading images, building a circle of friends and chatting online through specific social media services (Chen \& Chan, 2017; Pang, 2016; Zhong, 2014).

Moreover, research into social media usage patterns suggested that most of the college students are using MySpace and Facebook for an indispensable component of their everyday lives for reasons such as developing new friendships as well as re-connecting with old friends (Raacke \& Bonds-Raacke, 2008). Wang, Tchernev, and Solloway (2012) found similar results, indicating that four main motivations for social media use included emotional expression, cognition, social maintenance, and habitual diversion. In a study of 597 respondents, $\mathrm{Ku}, \mathrm{Chu}$, and Tseng (2013) discovered four general gratifications among the computer-mediated communication technologies, consisting of social maintenance, information exchange, amusement, and style. Based an online survey, Ruehl and Ingenhoff (2015) applied a combined perspective of uses and gratifications (U\&G) and social cognitive theory (SCT) to assess individuals' motives for brand page usage on Facebook, indicating that consumption behavior could be drived by activity, self-reactive-novel as well as monetary incentives. Later, Gan and Wang (2015) revealed that three patterns of gratifications were obtained from using both microblog and WeChat: content gratification, social gratification and hedonic gratification. In addition, they found that content gratification plays the most salient role in using microblog, while social gratification is the most important for WeChat usage. Although what motivates users to adopt social media and how the gratifications affect the practices through social media are crucial research subjects (Gan \& Wang, 2015; Leung, 2013; Quinn, 2016), prior published studies assessing the effects of these local social media platforms on in- dividual's behaviour in the Chinese society is scarce at best (Gan \& Wang, 2015). The aims of the current investigation are mainly to determine the social-psychological origins for college students to use these domestic social media, and to identify the gratifications they obtained from the media usage.

\section{Uses and gratifications theory}

$U \& G$, is one of the most established theories to explain the questions of "how" and "why" individuals actively choose specific media to gratify a variety of needs. As Katz suggested that U\&G theory is a useful approach in media research to understand the social and psychological origins of motives that affect people's purposely choose particular types of media (Katz, 1959). The key assumptions of the theory are on what individual do with the communication media instead of the influence of the specific media on the individual. It asserts that varied media compete for audiences' attention, and that users play an active role in selecting the medium that satisfies their distinctive types of needs, such as seeking out information, keeping emotional bonds, as well as chasing status (Valkenburg, Peter, \& Walther, 2016). A considerable amount of the prior research on $U \& G$ has paid attention to television (Nabi, Stitt, Halford, \& Finnerty, 2006), newspaper (Lin, Salwen, \& Abdulla, 2005) and other patterns of conventional media (Albarran et al., 2007). With the dynamic growth and popularization of new media technique, a large number of important studies from the U\&G perspective have appeared recently (Choi, 2016; Gan \& Wang, 2015; Jers, 2014; Verot, 2016). This theory is especially well-suited to unpacking social media platforms, which offer the potential for both mass and interpersonal communication (Lin, Hsu, Chen, \& Fang, 2017).

This study provides an empirical foundation for understanding what promptes social-network site users to adopt the new media platforms and what types of psychological needs these are offering. As Ha and James pointed that an essential dis- 
tinguishing nature of emerging media is interactivity, which enables users to offer information response to a source or communication from other users (Ha \& James, 1998). Similarly, Ruggiero identifies that concepts to be considered in the new media age, such as activity and interactivity, the essence and the characteristic of the online audience and asynchronies need to be reviewed (Ruggiero, 2000). As far as the nature of online communication is concerned, the differences become blur between consumer and producer, which has led to the use of the concept "prosumer" to explain users who are simultaneously involved in both production and consumption of content $(\mathrm{Ku}, \mathrm{Chu}, \&$ Tseng, 2013; Ritzer, 2010). This allows users control the message and its utilizaion, making it significant to measure the received gratifications through the media in the computer-mediated communication environment (Choi, 2016). As such, the internet and more specifically social media constitute newer media forms and new spread way, with their own defining characteristics in different countries, which are ripe for examination with the theoretic support of $U \& G$ theory.

\subsection{Gratifications sought on social media use}

In recent years, the theory of $U \& G$ has been reformulated to stress comparisons between the gratifications sought from a particular medium with gratifications obtained. Gratifications obtained refers to those different patterns of motives that individual's actual experience with the particular medium. However, gratifications sought means those particular gratifications that individual initially hope to get from a particular media before they have adopted the technology (Malik, Dhir, \& Nieminen, 2016). Palmgreen, Wenner, and Rayburn (1980) argued that while individuals purposely use media to meet certain types of needs, their needs are not always satisfied. Consumers tend to seek from using a special media that can offer the general and specific gratifications when a medium cannot fulfil their sought-after gratifications. This implies that Gratifica- tion obtained might predict the degree of users' satisfaction or dissatisfaction that their experience from using a certain medium (Palmgreen, Wenner, \& Rayburn, 1980). Therefore, it's important to also realize the gap between those two different dimensions of gratifications so that researchers have a clear idea of the analysis of how different consumers use various media applications in their daily life.

Considering social media is a novel social phenomenon in Mainland China, there is a distinct lack of empirical research in the gratifications or benefits of starting using the peculiar medium (Leung, 2013; Zhong, 2014). Meanwhile, only a few documents examined both gratifications obtained and motivations for using social media (Gan \& Wang, 2015; Pang, 2016; Quan-Haase \& Young, 2010). An examination of the motivations for using social media is necessary due to it would shed light into what factors impact widely adoption (Lin et al., 2017; Xiong \& Lv, 2013). Thus, this study proposes: what types of gratifications do individuals expect to get from a medium before using it? Are there one or multiple factors that drive them to embrace with such new media and tools? Thus, to address this important void in the literature, the first research question is proposed in the following:

, RQ1: How does the U\&G framework explain college students' local social media (especially QQ, WeChat and Weibo) adoption in Mainland China?

\subsection{Gratifications obtained from social media use}

Instead of only paying attention to the gratifications that people expect to receive from social media, this research applies the theory of uses and gratifications (U\&G) to comprehend the dimensions of gratifications that are obtained from their adoption of the services. As early as 1974, Blumler and Katz suggested that the majority of studies pay attention on gratifications obtained due to it helps us to understand what drives continued adoption of the media technologies (Blumler \& Katz, 1974). Numerous researchers have adopted U\&G 
framework to unpack the motivations for using of diversified social media platforms. Regarding IM usage, for instance, five gratifications sought are discovered, such as amusement, connection maintenance, freer expression, social interaction (Lo \& Leung, 2009). In terms of microblog adoption, Zhang and Pentina revealed that Chinese people seek several primary needs such as career development, feelings vent, information, citizenship behavior, social bonding, visibility, self-expression as well as keeping interaction (Zhang \& Pentina, 2012).

For using WeChat, Lien and Cao found individuals who have greater needs for recreation, information, and sociality are more willing to use WeChat. Furthermore, these three factors positively impact users' attitudes and trust (Lien \& Cao, 2014). Similarly, Li, Wang, Lu, Wang, and Zhu (2016) also revealed, for Chinese college WeChat users to use WeChat is mainly used to communicate and maintain the relationship between friends. Although these studies provided the preliminary explanation of the various and complex nature of gratifications that individuals obtain from using SNSs, only very few investigations have systematically analyzed the particular gratifications obtained from social media in the context of China (Wu, Shi, \& Yan, 2013). Therefore, based on the existing literature, the study attempts to probe the gratifications students get from using social media:

, RQ2: How do the different constructs extracted from the principal components factor analysis explain college students' domestic social media satisfaction in Mainland China?

\subsection{The association between the gratifications obtained and social media use}

Gratifications obtained are deemed as a crucial predictor of people's media use habits (Quan-Haase \& Young, 2010). As Palmgreen and Rayburn suggested that when a certain media satisfies the expected needs, this may result in the constant employ of the media (Palmgreen \& Ray- burn, 1979). In a study of Facebook, Joinson revealed that gender, view friends' photos, and frequency of state updates might predicte the number of users logging in the platform. Instead, age, as well as scores on the content gratification scale might predict the time spent on the website (Joinson, 2008). Wang, Tchernev, and Solloway (2012) found that social media use would significantly enhance emotional and cognitive aspects of gratifications, especially when these requirements are higher. In line with the study, Leung (2013) also revealed that people who use Facebook and blogs to satisfy their social and affection needs tend to spend more time using them. Recently, Khan (2017) found that specific social media behaviors such as commenting and uploading could be strongly predicted by social interaction need, while sharing through the technology being strongly predicted by informational need. Hence, in order to continue this line of studies, the following hypothesis was formulated:

RQ3: What is the relationship between the gratifications obtained and social media use?

\section{Methodology: Online survey}

\subsection{Sampling}

An online survey was undertaken at a Lanzhou university in Northwestern China in June and August 2016. The use of the university student sample is determined to be appropriate for the present study as they constitute the largest group of social media users (Gan, 2017). Students were invited to fill in an online questionnaire hosted on www.sojump.com about their use of three leading social media platforms in China including QQ, WeChat, and Weibo. Sojump is a very famous professional online survey and evaluation platform that provides humanized services consisting of online questionnaire design, data collection, custom reporting, and statistical analysis in China. Meanwhile, they were assured of anonymity and participation was entirely voluntary. The language was 
in Chinese and a total of 258 participants completed the online survey. When the non-users of social media were eliminated, the sample was reduced to 229 The study removed nonusers ( $11.2 \%)$ because the work presently concentrates on the gratifications derived from the utilization of social media. Of the sample, males comprised 67 percent of the sample and females 33 percent. Their average age was 23 years. The majority of them logged on to social media platforms more than five times every day $(n=153,66.8 \%)$ and spent more than two hours on social media daily $(\mathrm{n}=171,74.6 \%)$. In addition, the average monthly household income for the total sample in this study was about RMB 4500. It terms of education, $50.8 \%$ of the students holding the master degree, followed by $39.2 \%$ with the bachelor degree, $3.5 \%$ with the junior college degree and $6.5 \%$ doctoral degree.

\subsection{Constructs and measures}

The questionnaire was composed of ten questions concerning the use of social media, gratifications sought, gratifications obtained, and demographic characteristics. It took the participants on average 15 minutes to fill in the questionnaire. In order to ensure maximum response from these Chinese students, the questionnaire was translated into Chinese language by back translation method.

Social media behavior. Adopting past measures on social media use (Cheng, Liang, \& Leung, 2015; Gan \& Wang, 2015), this measure includes two self-reported assessments of social media behaviour, which were both treated as dependent variables in the regression models. The first test was used to examine the frequency of students' social media uses on an 8-point Likert scales, ranging from $1=1-2$ times to $5=$ more than nine times per day. The second assessment asked students how long they spent on social media each day on an 8-point scale ranging from $1=0-3$ hours to $5=$ more than 12 hours a day (see Table 1). In addition, to get an evaluation of students' social media uses experience, they were asked to report how long they had been adopting social media.
Table 1: Demographic characteristics of participants $(\mathrm{N}=229)$

\begin{tabular}{|c|c|}
\hline Demographic Characteristics & $\%$ \\
\hline \multicolumn{2}{|l|}{ Gender } \\
\hline Male & 67.2 \\
\hline Female & 32.8 \\
\hline \multicolumn{2}{|l|}{ Age } \\
\hline Under 20 & 4.8 \\
\hline $20-25$ & 59.8 \\
\hline $26-31$ & 24.0 \\
\hline Above 31 & 11.4 \\
\hline \multicolumn{2}{|l|}{ Education } \\
\hline College & 1.7 \\
\hline Bachelor & 53.3 \\
\hline Master & 38.4 \\
\hline Doctoral & 6.6 \\
\hline \multicolumn{2}{|l|}{ Income } \\
\hline Below $3000 \mathrm{RMB}$ & 29.3 \\
\hline 3000-6000 RMB & 41.0 \\
\hline $6001-9000 \mathrm{RMB}$ & 14.8 \\
\hline 9001-12000 RMB & 7.4 \\
\hline More than $12001 \mathrm{RMB}$ & 7.4 \\
\hline \multicolumn{2}{|l|}{ Frequency use of social media } \\
\hline $1-2$ times & 14.8 \\
\hline 3-4 times & 18.3 \\
\hline $5-6$ times & 16.2 \\
\hline 7-8 times & 9.6 \\
\hline More than 9 times & 41.0 \\
\hline \multicolumn{2}{|l|}{ Time spend on social media } \\
\hline $0-3$ hours & 75.5 \\
\hline $3-6$ hours & 16.6 \\
\hline $6-9$ hours & 5.2 \\
\hline 9-12 hours & 1.9 \\
\hline More than 12 hours & 0.9 \\
\hline
\end{tabular}

Gratifications sought. To assess motivations or reasons why college students use social media, the study measured gratifications sought by using the scale of gratifications sought propounded by QuanHaase and Young (Quan-Haase \& Young, 2010). The items are fit within the present research due to Instant Messaging and Facebook are mainly used for social needs, hence, similar gratifications sought are expected to drive students' social media use in China. The scale totally consisted of 11 relevant statements, which comprised a variety of motivations that survey respondents could select from. Respondents were required to report whether they use social 
networking sites for these reasons on yes or no scale with " 1 " =yes and "0" = no (see Table 2).

Table 2: Gratifications sought in percentages ( $N=229$ )

\begin{tabular}{lr}
\hline Individual Items & \% of Yes responses \\
\hline Friend suggested it & 45.8 \\
Everyone I know is on social media & 90.3 \\
Help others keep in touch with me & 96.5 \\
Find classmates & 80.0 \\
Received a promotional e-mail & 55.0 \\
Get to know more people & 43.2 \\
Network in general & 82.0 \\
Find course information & 73.3 \\
Find dates & 22.2 \\
Find people with mutual interests & 44.5 \\
Find jobs & 24.4 \\
\hline Note. Percentage of sample in the questionnaire who responded \\
"yes" to the item.
\end{tabular}

Gratifications obtained. Gratifications obtained assessed the range of benefits people received from their use of social media sites. Based on $\mathrm{Ku}$, Chu, and Tseng's (2013) study, the study adopted 26 items to assess respondents' motivations for using social media the respondents were asked, "I use social media to...?" and they could rate each statement on a five-point response scale ranging from strongly disagree (1) to strongly agree (5). Table 3 shows wording, means, as well as standard deviations for each of the item. The items used by $\mathrm{Ku}$, Chu, and Tseng are suitable for the current research because, the elements employed in this study resulted from integrating statements from prior literature indicated main gratifications from adopting the new media technologies (Lo \& Leung, 2009; Wang, Tchernev, Solloway, 2012). Control measures Demographic attributes of study participants were measured by age, gender, overall monthly family income and education level. Background information was gathered for each student to offer an overview of demographic characteristics in the analysis.

\subsection{Data analysis}

The data analysis was performed by using SPSS 20.0. Firstly, a descriptive analysis was performed on the data to examine the psychological incentives of using social media. Secondly, a principal components factor analysis with varimax rotation was conducted to gauge the types of gratifications obtained from social media usage. Based on the findings of the factor loadings in the study, items means, standard deviations (SD), and the standardised Cronbach's alpha, were computed for every factor. Thirdly, ordinary least squares regression analyses were run to test the association between gratifications obtained and students' social media usage behaviors.

\section{Results}

\subsection{Social media use}

In the survey, $41 \%$ stated visiting their social media more than nine times per day. $14.8 \%$ respondents launch their social media account at least once or twice per day, $18.3 \%$ use it at least three or four times a day, $16.2 \%$ update it at least five or six times a day, and $9.6 \%$ update it seven or eight times per day. The data shows that the participants use social media extensively, logging into their accounts to send messages, browse photos, check the recent status of their friends, as well as get interesting content on upcoming social issues. Regarding spending time on social media, on an average day, the study found that approximately three-quarters of student spent 0-3 hours and more than a quarter of student spent more than three hours on these sites. Besides, data showed also that a majority of the students $(86.0 \%)$ had been using social networking sites over two years $(M=4.79, S D=0.59)$.

\subsection{Gratifications-sought for using social media}

Of participants surveyed, 96.5 percent of them claimed that their main gratifications for using social media were that "help others keep in touch with me" (see Table 2). This result is consistent with the 
Table 3: Factor analysis for social media gratifications

\begin{tabular}{|c|c|c|c|c|c|c|c|}
\hline \multirow[t]{2}{*}{ I use social media... } & \multirow[t]{2}{*}{ M } & \multirow[t]{2}{*}{ SD } & \multicolumn{5}{|c|}{ Factors } \\
\hline & & & 1 & 2 & 3 & 4 & 5 \\
\hline \multicolumn{8}{|l|}{ Relationship maintenance } \\
\hline To tell friends and family I am all right & 3.64 & 0.900 & 0.738 & 0.118 & 0.099 & 0.130 & 0.100 \\
\hline To let others know I care for them & 3.61 & 0.875 & 0.739 & 0.088 & 0.145 & 0.207 & 0.186 \\
\hline To keep in contact with family and friends & 4.03 & 0.802 & 0.794 & 0.160 & -0.057 & 0.117 & 0.048 \\
\hline To feel closer to my family and friends & 3.71 & 0.891 & 0.810 & 0.213 & 0.153 & 0.174 & 0.023 \\
\hline To improve the relationship with my family and friends & 3.62 & 0.828 & 0.715 & 0.127 & 0.243 & 0.207 & 0.087 \\
\hline To organize social events & 3.87 & 0.778 & 0.650 & 0.197 & 0.052 & 0.087 & 0.215 \\
\hline To keep in contact with people I have no time to meet & 4.03 & 0.688 & 0.728 & 0.149 & 0.005 & 0.057 & 0.174 \\
\hline To interact with my family and friends easily & 4.06 & 0.629 & 0.757 & 0.238 & 0.060 & 0.015 & 0.100 \\
\hline \multicolumn{8}{|l|}{ Amusement } \\
\hline Because it is entertaining for me & 3.73 & 0.781 & 0.173 & 0.666 & 0.018 & 0.228 & 0.378 \\
\hline Because it is pleasant & 3.74 & 0.732 & 0.285 & 0.813 & 0.072 & 0.167 & 0.212 \\
\hline Because it relaxes me & 3.78 & 0.736 & 0.316 & 0.803 & 0.132 & 0.243 & 0.142 \\
\hline To have fun & 3.65 & 0.790 & 0.225 & 0.791 & 0.193 & 0.231 & 0.200 \\
\hline To enjoy the pleasure of contacting people & 3.71 & 0.759 & 0.431 & 0.588 & 0.161 & 0.215 & 0.145 \\
\hline \multicolumn{8}{|l|}{ Style } \\
\hline To feel that I am a grown-up person & 3.26 & 0.828 & 0.361 & 0.271 & 0.526 & 0.160 & 0.092 \\
\hline As a status symbol & 2.69 & 0.984 & 0.145 & 0.032 & 0.861 & 0.065 & 0.078 \\
\hline To look stylish & 2.76 & 0.977 & 0.031 & 0.093 & 0.891 & 0.173 & 0.197 \\
\hline To look fashionable & 2.75 & 0.948 & 0.025 & 0.107 & 0.892 & 0.169 & 0.213 \\
\hline \multicolumn{8}{|l|}{ Information seeking } \\
\hline To find out new information for myself & 3.55 & 0.855 & 0.226 & 0.172 & 0.306 & 0.727 & 0.093 \\
\hline To obtain useful information & 3.60 & 0.824 & 0.211 & 0.230 & 0.216 & 0.823 & 0.153 \\
\hline To obtain helpful information & 3.68 & 0.800 & 0.201 & 0.182 & 0.182 & 0.867 & 0.093 \\
\hline To acquire information in an inexpensive way & 3.78 & 0.798 & 0.121 & 0.326 & -0.036 & 0.679 & 0.250 \\
\hline \multicolumn{8}{|l|}{ Sociability } \\
\hline To meet new acquaintances & 3.42 & 0.863 & 0.210 & 0.099 & 0.349 & 0.220 & 0.605 \\
\hline To be less inhibited chatting with strangers & 3.19 & 0.966 & 0.107 & 0.089 & 0.420 & 0.166 & 0.514 \\
\hline To allow people to find me easily & 3.43 & 0.879 & 0.270 & 0.136 & 0.168 & 0.186 & 0.623 \\
\hline To relieve boredom by contacting people & 3.53 & 0.891 & 0.062 & 0.250 & 0.043 & 0.060 & 0.779 \\
\hline To joke with friends & 3.55 & 0.819 & 0.159 & 0.230 & 0.106 & 0.047 & 0.811 \\
\hline Eigenvalue & & & 10.271 & 2.874 & 2.027 & 1.653 & 1.153 \\
\hline$\%$ of variance & & & 20.459 & 13.134 & 12.815 & 11.606 & 11.137 \\
\hline Cronbach's alpha & & & 0.909 & 0.907 & 0.878 & 0.884 & 0.810 \\
\hline
\end{tabular}

previous literature, which also argued that majority of people use the virtual communities in order to obtain timely communication and keep the connection with each other (Ifinedo, 2016). Once a person has logged in a social media account, he or she may feel a need to interact with their friends through it and hence recommend it to other people. This also confirms the previous virtual community studies in the diffusion of innovations tradition, indicating that information regarding an innovation frequently comes via personal social networks such as family members, friends, or colleagues (Rogers, 2010). The second need chosen frequently by $90.3 \%$ was "everyone I know is on social media." Considering that students taped these social media sites to meet some new friends and decrease social inhibitions (Pang, 2016), social media is very popular among students and its adoption leads to a need to be part of the group. Conversely, not using social media like others would imply 
being excluded from the circle of friends and acquaintances. The third statement students selected most often $(82.0 \%)$ was "network in general." The item followed by "find classmates" with $80.0 \%$. These two statements indicate a requirement for social connectivity that enables young people to stay loosely connected with their friends and family members and to become the member of the larger social media community. However, only $22.2 \%$ of users make use of social media to search for potential dates. Table 2 displays that other motivations were selected much less often by students as reasons for using social media.

\subsection{Gratifications obtained from social media use}

When exploring gratifications obtained from using social media, six factors were determined with the eigenvalues greater than 1.0, explaining $69.15 \%$ of the variance completely (see Table 3). Factor 1, relationship maintenance, consists of eight items measuring how social media provides a venue for the formation and maintenance of the connection with others (eigenvalue $=10.271$, variance explained $=20.459 \%$ ) .

Factor 2, pastime, consists of five items, all of which were related to college students' use of social media as a mean for receive entertainment ends such as relaxing, having fun and getting enjoyment. The eigenvalue is 2.874 , and the factor explains $13.134 \%$ of the corresponding percentages of variance. The mean for major of these statements was high, representing users' key gratifications with respect to social media use. Two significant motivations were "because it relaxes me" $(M=3.78, S D=0.736)$ and "because it is pleasant" $(M=3.74, S D=0.732)$, indicating how university students perceive social media as a vehicle to seek to relax and enjoy themselves.

Ranked as the third factor, style (eigenvalue $=2.027, \quad$ variance explained $=12.815 \%$ ) concludes three items assessing the extent to which social media enables users to look really fashionable and stylish. Factor 4, information seeking (eigenvalue $=1.653$, variance explained $=11.606 \%$ ), consists of four items measuring the extent to which users would like to know what's happening to the latest issues and events. The high mean implies that for individuals, being in the "social know" is vital due to social information is necessary to become a member of the peer group. The last construct considered was the sociability entertainment motivation (eigenvalue $=1.153$, variance explained $=11.137 \%$ ), which contains three items that tests the students' willingness to utilize social media to get to know new people and reduce social inhibitions and boredom.

\subsection{Gratifications obtained and social media use}

How are gratifications obtained and social media use associated with each other? Two ordinary least squares regression models test were conducted to systematically examine the connection between the five gratification factors and social media usage (see Table 4). The first model consisted of the frequency of social media use and the second model included the time spend on social media as the dependent variable. As shown in Table 4, regression model 1 reveals that there are significant correlations between education and the frequency of social media usage. The lower grade students tend to log onto the services more frequently in a day. It is possible that the job pressure of senior students was higher than that of junior students in the university (Vromen, Loader, Xenos, \& Bailo, 2016). In this model, when controlling for the influence of the population variables, the study finds that both relationship maintenance $(\beta=0.159, p<0.05)$ and amusement $(\beta=0.131, p<0.05)$ are positively associated with the frequency of social media use. This model makes up $6.0 \%$ of the variance in the frequency of social media use in model 1 .

The second regression model indicates no relationship of demographics variable with students' spending time on social media. After gender, age, education and income were controlled in the model, sociability $(\beta=0.193, p<0.01)$ are posi- 
tively associated with time spent on social media. Therefore, the student spent more time on social media per session are mainly to fulfil their sociability needs. However, it is interesting to notice that style $(\beta=$ $-0.131, p<0.05$ ) are negatively associated with time spent on social media. The independent variables demonstrate an entire of $6.0 \%$ of the variance in updating profiles. Thus it can be seen that the gratifications obtained factor explain more variance in the profile updates than the frequency of social media use.

Table 4: $\quad$ Regression analysis of social media use ( $N=229$ )

\begin{tabular}{lcc}
\hline Variables & $\begin{array}{c}\text { Frequency of } \\
\text { social media use } \\
\beta\end{array}$ & $\begin{array}{c}\text { Time spend on } \\
\text { social media } \\
\beta\end{array}$ \\
\hline Demographics & & \\
Age & 0.022 & 0.064 \\
Gender(male) & 0.079 & -0.149 \\
Education & $-0.179^{*}$ & 0.056 \\
Income & -0.090 & 0.123 \\
Gratifications & & \\
Relationship & & \\
maintenance & $0.159^{*}$ & 0.105 \\
Amusement & $0.131^{*}$ & 0.035 \\
Style & -0.054 & $-0.131^{*}$ \\
Information seeking & 0.070 & -0.079 \\
Sociability & 0.109 & $0.193^{* *}$ \\
Adjusted $R^{2}$ & $0.060^{* *}$ & $0.060^{* *}$
\end{tabular}

Note. OLS $=$ ordinary least squares. Table depicts standardized coefficients from regression models 1 and $2 .{ }^{*} p<0.05 .{ }^{* *} p<0.01$.

\section{Conclusions and discussion}

\subsection{Major findings}

The rapid development of social media has attracted tremendous academic attention, leading to the widespread investigations concentrating on the uses and possible implications of those new technologies. However, much of the research to date only paid attention to social media sites in western countries, neglecting to investigate the widespread domestic social media use among university students in Asian countries. Thus, the present study attempts to fill this void by applying the uses and gratifications framework to explore both the behaviors of gratifications sought and gratifications obtained in the use of leading local social media technologies among college student in the Chinese society. It is expected that the exploratory work not only brings new insight to the U\&G framework, but also offers firsthand information on the status of domestic social media use in Mainland China.

Firstly, the results identify students' the underlying types of gratifications that are sought from using social media. Among them, "everyone I know is on social media," "help others keep in touch with me," and "network in general" rank as the principal motives that drive students' choice of local technologies. The findings indicate that the main socio-psychological needs for adopting domestic social media are to get instant communication as well as maintain interaction with the peer network. These findings are consistent with similar research by Quan-Haase and Young that found the peer network not only provides a series of content relevant with the innovation, but also generates social pressure for the adolescent to adopt (QuanHaase \& Young, 2010). The empirical work confirms that individuals are seeking for more convenient and high-efficient communication ways to keep connections with others via a variety of media, especially when these media is much easier to get and operate (Cheng, Liang, \& Leung, 2015). The result also reinforces Phua, Jin, and Kim's (2017) quantitative study that revealed social innovations might diffuse through people's social bonds with one another.

Secondly, factor analysis successfully generated the five-factor structure of the gratifications obtained among social media users. For the exploratory procedures, the study selected principal component analysis instead of maximum-likelihood factor analysis, not only because the hypotheses were themselves exploratory, but also because the aim of study was to identify a few possible coherent constructs that best reflected the various meaningful dimensions of motivations. As some scholar suggested that principal com- 
ponent analysis could obtain the most information content about the specific substrates tested in the research, thereby, the results from it would offer a best-case scenario for the linear combination metric (Shankarraman, Davis-Gorman, Copeland, Caplan, \& McDonagh, 2012). Results show that students are motivated by relationship maintenance needs, the purpose for amusement, chasing style, seeking information, and sociability needs. This result may reflect individuals are mainly motivated to utilize social media services for the purpose of seeking relationship maintenance and entertainment, thereafter for their inner social and psychological needs. Leung also found the same finding and further suggested that social networking applications are ideal platforms for people to keep a large network base of superficial connections, such as virtual friends through the computer-mediated technologies (Leung, 2013). The entertainmental need is the second discovered gratification among the uses and gratifications test. This result is also supported by related research (Cheng, Liang, \& Leung, 2015; Ifinedo, 2016; Zhong, 2014). Given that social media is a multimedia tool, using the service to read useful information as well as playing online games, may be more attractive for young adults when pursuing amusement than traditional medium. Moreover, consistent with previous investigations (Cheng, Liang, \& Leung, 2015), this study finds that style is an integral and indispensable factor of social media usage in Chinese society.

Thirdly, this study investigated gratification predictors for the frequency of social media use and the time spent in it. When the study controlled demographic variables, social media usage is predicted by relationship maintenance and amusement. The findings indicate that those with high desire to stay in touch with friends and enjoy the entertainment online tend to use social media more often. These findings echo the work by Fu's group who discovered that people who actively use social media because of inbred psychological incentives to keep the connection with distand and closed friends that they can satisfy their needs $(\mathrm{Fu}, \mathrm{Wu}$, \& Cho, 2017). Meanwhile, this empirical results also support the general view that through the social media usage process, the young adult cohort has the opportunity to socialize with their friends, and be entertained (Choi, 2016; Leung, 2013; Lien \& Cao, 2014).

\subsection{Implications for research and practice}

Results of the current study provide both academic and practical implications. First, the present research makes theoretical contributions to existing literature on $U \& G$ (Choi, 2016; Ji, 2017; Vromen et al., 2016) by adopting the U\&G approach to explore the psychological incentives and domestic social media behaviors among university students in the Chinese setting. Second, this article identifies five key gratifications behind the use of these web-based technologies, and further indicates social media use could be strongly predicted by certain psychological incentives such as social interaction and amusement. Third, this research offers a wealth of information in guiding relevant practitioners in developing ideal social media strategies where social networking sites are crucial components of the media market (Whiting \& Williams, 2013). Moreover, appropriately considering the determined gratifications while generating future related functions and uses, social media services practitioners can make the social media platforms more attractive for users.

\subsection{Limitations and future work direction}

In explaining the findings of the current research, one must focus on some limitations of the exploratory research that offers directions for future studies. Firstly, due to the relatively modest sample size in the research recruited through online, so the results cannot be generalized to the larger population of social media users. Meanwhile, this study examines social media users' motivations, use behaviors in the Chinese context, hence the findings may not be generalized to social media users in other settings. Secondly, 
the study respondents were mostly young users of domestic social media such like QQ, WeChat, and Weibo. Care must be taken when applying the results to other patterns of social media platforms that target different populations of users. Thirdly, due to the cross-sectional nature of the present research, it thus may limit causality among the various variables of theoretical interest (Ran, Yamamoto, \& Xu, 2016). Although the research assumed that media use behaviors would lead to satisfying respondents' psychological needs, the opposite directionality is equally plausible. It is likely that people who deem they enable to meet their gratifications by using the new services, they are likely to access the media more often (Fu, Wu, \& Cho, 2017). To draw such precise causal inferences, a longitudinal analysis is needed in the near future. Lastly, future studies should also focus on other media usage behaviors, and the role of distinct gratifications in impacting individuals' usage patterns.

Despite the limitations, this research originally offers a much-needed investigation of gratifications soughts, gratifications obtained and domestic social media use behaviors among Chinese university students based on the uses and gratifications framework. This paper demonstrates the significance and valuable of uses and gratifications theory to social media studies in Asian countries. The results further suggest that the rapid rise of domestic social media technologies in contemporary China have in many ways afforded young people more opportunities to connect the social life and satisfy a wide range of needs that were impossible a decade before. Therefore, it is expected that the current research could trigger both theorizing and empirical studies on the new media technologies in the current media environment.

\section{References}

Albarran, A. B., Horst, J. L., Khalaf, T., Lay, J. P., McCracken, M., Mott, B. ... \& Guo, M. (2007). "What happened to our audience?" Radio and new technology uses and grat- ifications among young adult users. Journal of Radio Studies, 14(2), 92-101.

Blumler, J. G., \& Katz, E. (1974). The uses of mass communications: Current perspectives on gratifications research. Beverly Hills, CA: Sage Publications.

Chen, Z., \& Chan, M. (2017). Motivations for social media use and impact on political participation in China: A cognitive and communication mediation approach. Cyberpsychology, Behavior, and Social Networking, 20(2), 83-90.

Cheng, Y., Liang, J., \& Leung, L. (2015). Social network service use on mobile devices: An examination of gratifications, civic attitudes and civic engagement in China. New Media \& Society, 17(7), 1096-1116.

Choi, J. (2016). Why do people use news differently on SNSs? An investigation of the role of motivations, media repertoires, and technology cluster on citizens' news-related activities. Computers in Human Behavior, 54, 249-256.

Fu, P.-W., Wu, C.-C., \& Cho, Y.-J. (2017). What makes users share content on facebook? Compatibility among psychological incentive, social capital focus, and content type. Computers in Human Behavior, 67, 23-32.

Gan, C. (2017). Understanding WeChat users' liking behavior: An empirical study in China. Computers in Human Behavior, 68, 30-39.

Gan, C., \&Wang, W. (2015). Uses and gratifications of social media: A comparison of microblog and WeChat. Journal of Systems and Information Technology, 17(4), 351-363.

Ha, L., \& James, E. L. (1998). Interactivity reexamined: A baseline analysis of early business web sites. Journal of Broadcasting \& Electronic Media, 42(4), 457-474.

Hu, T., Marchiori, E., Kalbaska, N., \& Cantoni, L. (2014). Online representation of Switzerland as a tourism destination: An exploratory research on a Chinese microblogging platform. Studies in Communication Sciences, 14(2), 136-143.

Huang, R., Kim, H., \& Kim, J. (2013). Social capital in QQ China: Impacts on virtual engagement of information seeking, interaction sharing, knowledge creating, and purchasing intention. Journal of Marketing Management, 29(3-4), 292-316. 
Ifinedo, P. (2016). Applying uses and gratifications theory and social influence processes to understand students' pervasive adoption of social networking sites: Perspectives from the Americas. International Journal of Information Management, 36(2), 192-206.

Jers, C. (2014). Konsumieren, partizipieren und produzieren im Web 2.0: Ein sozial-kognitives Modell zur Erklärung der Nutzungsaktivität. Köln, Germany: Herbert von Halem Verlag.

Ji, Q. (2017). Social media news use and political discussion: A focus on Chinese users' news reception and dissemination. Electronic News, 11(1), 3-19.

Joinson, A. N. (2008). Looking at, looking up or keeping up with people?: Motives and use of facebook. Paper presented at the Proceedings of the SIGCHI conference on Human Factors in Computing Systems, Florence, Italy.

Kammerl, R., \& Kramer, M. (2016). The changing media environment and its impact on socialization processes in families. Studies in Communication Sciences, 16(1), 21-27.

Katz, E. (1959). Mass communications research and the study of popular culture: An editorial note on a possible future for this journal. Studies in Public Communication, 2, $1-6$.

Khan, M.L. (2017). Social media engagement: What motivates user participation and consumption on YouTube? Computers in Human Behavior, 66, 236-247.

Ku, Y.-C., Chu, T.-H., \& Tseng, C.-H. (2013). Gratifications for using CMC technologies: A comparison among SNS, IM, and e-mail. Computers in Human Behavior, 29(1), 226-234.

Leung, L. (2013). Generational differences in content generation in social media: The roles of the gratifications sought and of narcissism. Computers in Human Behavior, 29(3), 997-1006.

Li, Y., Wang, R., Lu, X., Wang, X., \& Zhu, J. (2016). Report and investigation model of WeChat popularization in China's colleges and universities in 2015. Paper presented at the International Conference on Intelligent Control and Computer Application (ICCA 2016), Zhengzhou, China.
Lien, C. H., \& Cao, Y. (2014). Examining WeChat users' motivations, trust, attitudes, and positive word-of-mouth: Evidence from China. Computers in Human Behavior, 41, 104-111.

Lin, C., Salwen, M. B., \& Abdulla, R.A. (2005). Uses and gratifications of online and offline news: New wine in an old bottle. Online news and the public, 221-236.

Lin, Y.-H., Hsu, C.-L., Chen, M.-F., \& Fang, C.-H. (2017). New gratifications for social wordof-mouth spread via mobile SNSs: Uses and gratifications approach with a perspective of media technology. Telematics and Informatics, 34(4), 382-397.

Linkfluence. (2016). Top 10 Chinese social media sites. Retrieved March $15^{\text {th }}, 2016$, from http://linkfluence.com/en/2016/02/08/ top-10-chinese-social-media-sites/.

Lo, O.W.-Y., \& Leung, L. (2009). Effects of gratification-opportunities and gratifications-obtained on preferences of instant messaging and e-mail among college students. Telematics and Informatics, 26(2), 156-166.

Malik, A., Dhir, A., \& Nieminen, M. (2016). Uses and gratifications of digital photo sharing on Facebook. Telematics and Informatics, 33(1), 129-138.

Nabi, R. L., Stitt, C. R., Halford, J., \& Finnerty, K. L. (2006). Emotional and cognitive predictors of the enjoyment of reality-based and fictional television programming: An elaboration of the uses and gratifications perspective. Media Psychology, 8(4), 421-447.

Palmgreen, P., \& Rayburn, J. D. (1979). Uses and gratifications and exposure to public television a discrepancy approach. Communication Research, 6(2), 155-179.

Palmgreen, P., Wenner, L.A., \& Rayburn, J. D. (1980). Relations between gratifications sought and obtained a study of television news. Communication Research, 7(2), 161-192.

Pang, H. (2016). Understanding key factors affecting young people's WeChat usage: An empirical study from uses and gratifications perspective. International Journal of Web Based Communities, 12(3), 262-278.

Phua, J., Jin, S.V., \& Kim, J. J. (2017). Uses and gratifications of social networking sites for bridging and bonding social capital: A comparison of Facebook, Twitter, Insta- 
gram, and Snapchat. Computers in Human Behavior, 72, 115-122.

Quan-Haase, A., \& Young, A. L. (2010). Uses and gratifications of social media: A comparison of Facebook and Instant Messaging. Bulletin of Science, Technology \& Society, 30(5), 350-361.

Quinn, K. (2016). Why we share: A uses and gratifications approach to privacy regulation in social media use. Journal of Broadcasting \& Electronic Media, 60(1), 61-86.

Raacke, J., \& Bonds-Raacke, J. (2008). MySpace and Facebook: Applying the uses and gratifications theory to exploring friend-networking sites. Cyberpsychology \& Behavior, 11(2), 169-174.

Ragnedda, M. (2015). Electronic surveillance on Social Networking Sites. A critical case study of the usage of SNSs by students in Sassari, Italy. Studies in Communication Sciences, 15(2), 221-228.

Ran, W., Yamamoto, M., \& Xu, S. (2016). Media multitasking during political news consumption: A relationship with factual and subjective political knowledge. Computers in Human Behavior, 56, 352-359.

Ritzer, G. (2010). Prosumer revisited Focusing on the prosumer: On correcting an error in the history of social theory. (pp. 61-79). Wiesbaden, Germany: Verlag für Sozialwissenschaften.

Rogers, E. M. (2010). Diffusion of innovations. New York, America: Simon and Schuster.

Rosengren, K. E. (1974). Uses and gratifications: A paradigm outlined. The uses of mass communications: Current perspectives on gratifications research, 3, 269-286.

Ruehl, C. H., \& Ingenhoff, D. (2015). Communication management on social networking sites: Stakeholder motives and usage types of corporate Facebook, Twitter and YouTube pages. Journal of Communication Management, 19(3), 288-302.

Ruggiero, T.E. (2000). Uses and gratifications theory in the 21st century. Mass Communication \& Society, 3(1), 3-37.

Shankarraman, V., Davis-Gorman, G., Copeland, J. G., Caplan, M. R., \& McDonagh, P. F. (2012). Standardized methods to quantify thrombogenicity of blood-contacting materials via thromboelastography. Journal of Biomedical Materials Research Part B: Applied Biomaterials, 100(1), 230-238.
Statista. (2015). Number of social network users in selected countries in 2014 and 2018 (in millions). Retrieved March $15^{\text {th }}$ 2016, from http://www.statista.com/ statistics/278341/number-of-social-network-users-in-selected-countries/.

Sullivan, J. (2014). China's Weibo: Is faster different? New Media \& Society, 16(1), 24-37.

Valkenburg, P. M., Peter, J., \& Walther, J. B. (2016). Media effects: Theory and research. Annual Review of Psychology, 67, 315-338.

Verot, O. (2016). The 5 hottest Chinese social media trends for 2015. Retrieved March $15^{\text {th }}, 2016$, from http://maximizesocialbusiness.com/5-hottest-chinese-social-media-trends-china-2015-22427/.

Vorderer, P., Steen, F. F., \& Chan, E. (2006). Motivation. In B. Jennings \& P. Vorderer (Eds.), The psychology of entertainment (pp. 3-17). Mahwah, NJ: Lawrence Erlbaum.

Vromen, A., Loader, B. D., Xenos, M.A., \& Bailo, F. (2016). Everyday making through Facebook engagement: Young citizens' political interactions in Australia, the United Kingdom and the United States. Political Studies, 64(3), 513-533.

Wang, Z., Tchernev, J. M., \& Solloway, T. (2012). A dynamic longitudinal examination of social media use, needs, and gratifications among college students. Computers in Human Behavior, 28(5), 1829-1839.

Whiting, A., \& Williams, D. (2013). Why people use social media: A uses and gratifications approach. Qualitative Market Research: An International Journal, 16(4), 362-369.

Wu, S., Shi, Y., \& Yan, Y. (2013). Media exposure and Chinese cultural identity. Studies in Communication Sciences, 13(2), 129-132.

Xiong, C., \& Lv, Y. (2013). Social network service and social development in China. Studies in Communication Sciences, 13(2), 133-138.

Zhang, L., \& Pentina, I. (2012). Motivations and usage patterns of Weibo. Cyberpsychology, Behavior, and Social Networking, 15(6), 312-317.

Zhong, Z.-J. (2014). Civic engagement among educated Chinese youth: The role of SNS (Social Networking Services), bonding and bridging social capital. Computers \& Education, 75, 263-273. 